\title{
A rare association of Graves' disease and sarcoidosis
}

\author{
Ilyas El Kassimi ${ }^{1 *}$, Adil Rkiouak ${ }^{1}$, Nawal Sahel ${ }^{1}$, Meryem Zaizaa ${ }^{1}$, Youssef Sekkach ${ }^{1}$ \\ ${ }^{1}$ Internal Medicine Department, Mohammed V Military Teaching Hospital, Mohammed V University, Rabat, Morocco.
}

Graves' disease is the most common autoimmune disease in hyperthyroidism. Sarcoidosis is an inflammatory granulomatous disease of unknown cause. The association of the two diseases has rarely been reported in the literature. We report a new case of newly diagnosed sarcoidosis in a patient followed for Graves' disease. This is an original case of Graves' disease, the course of which was marked by the onset of active sarcoidosis presented as Löfgren's syndrome complicated by hypercalcemia. Given its rarity and the lack of a causal link, the association between Graves' disease and sarcoidosis may be mere coincidence. More studies could allow us to understand more common etiopathogenic mechanisms since both are chronic inflammatory diseases.

Keywords: Graves' disease; sarcoidosis

\section{Introduction}

Graves' disease is the most common autoimmune disease in hyperthyroidism. Sarcoidosis is an inflammatory granulomatous disease of unknown cause. The association of the two diseases has rarely been reported in the literature. Herein is reported a new case of newly diagnosed sarcoidosis in a patient followed for Graves' disease.

A 59-year-old woman was admitted for inflammatory polyarthralgia; she was a known hypertensive under amlodipine and had been followed for 4 years for Graves' disease under carbimazole. Her symptoms worsened rapidly over the three weeks after her admission with the onset of fever, deterioration of general condition, vomiting, and hyperalgesic erythematosus nodules on the legs which spontaneously disappeared. Examination revealed bilateral proptosis, goiter, joint pain predominantly in the large joints and arthritis in both ankles. The biological assessment showed hypercalcemia at $117 \mathrm{mg} / \mathrm{l}(\mathrm{NR}=90$ $-105 \mathrm{mg} / \mathrm{l}$ ) with normal a calciuria level. Thyroid function tests confirmed hyperthyroidism associated with a moderate inflammatory syndrome, C-reactive protein at $55 \mathrm{mg} / \mathrm{l}$ (Normal rate: $<6$ ), and sedimentation rate at $65 \mathrm{~mm}$ in the first hour (Normal rate : <30). Parathyroid hormone (PTH) and angiotensin converting enzyme (ACE) levelswere normal. Protein electrophoresis showed polyclonal hypergammaglobulinemia. The levels of lactate dehydrogenase (LDH) and $\beta 2$ microglobulin were normal. Cervical ultrasound and thyroid scintigraphy showed the appearance of Graves' disease. Chest X-ray and CT revealed non-compressive bilateral hilar mediastinal lymphadenopathy, suggesting stage I sarcoidosis (Figure 1). The tuberculin cutaneous and QuantiFERON tests were both negative as was the sputum test for tuberculosis. Bronchial fibroscopy showed a slightly inflammatory appearance of bronchial mucosa surface. The histological study of the bronchial biopsy showed a noncaseating epithelioid and gigantocellular granuloma (Figure 2) which may be consistent with sarcoidosis. The results of the histological study, the presence of typical hilar lymphadenopathy, polyclonal hypergammaglobulinemia, and hypercalcemia were strong arguments to conclude that it is sarcoidosis and to rule out other differential diagnoses, in particular infectious diseases such as tuberculosis or tumors such as lymphomas. In addition to the urgent conventional treatment of hypercalcemia with good enteral and parenteral hydration, the introduction of corticosteroid therapy (prednisone at a dose of $0.5 \mathrm{mg} /$ day) with a gradual decrease associated with potassium supplementation and the adjustment of the carbimazole dose made it possible to significantly improve the patient's condition with a relative regression of the exophthalmos.

Graves' disease is known to be associated with other autoimmune pathologies. Sarcoidosis also coexists with autoimmune diseases. Sarcoidosis is an inflammatory disease of unknown cause that affects one or more organs. The histopathologic hallmark of the disease is noncaseating granulomas. It usually presents in adults in their third through fifth decades [1]. Graves' disease is an autoimmune disease in which activated $\mathrm{T}$ and $\mathrm{B}$ cells may

Personal non-commercial use only. Rheumatology Research Journal. Copyright (C 2020. All rights reserved

*Corresponding Author: Ilyas El Kassimi: M.D, Internal Medicine Department, Mohammed V Military teaching Hospital, Mohammed V University, Souissi, Rabat, Morocco, E-mail: dr.elkassimiilyas@gmail.com,.

Received: 30 November 2019; Accepted: 04 January 2020 
lead to intrathyroidal lymphocytic infiltrations. Thyroid sarcoidosis has been rarely described, because it is generally asymptomatic, discovered by chance on an anatomopathological study of part of a thyroid resection. A literature review revealed that most studies sought to assess the prevalence of dysthyroidism in patients with sarcoidosis, not the reverse, and reported an incidence of autoimmune thyroid disease in patients with sarcoidosis. In a few cases, sarcoidosis has been codiagnosed with goiter and/or thyroiditis [2]. Gentilucci's study found that significant elevated antithyroglobulin autoantibody levels have been reported in patients with sarcoidosis [3]. A Japanese study reported that the prevalence of sarcoidosis related with Hashimoto's thyroiditis was 3-11\%, much more common than other thyroid diseases [4]. Ilias et al. were also interested in screening for thyroid disorders in patients followed for sarcoidosis. They concluded that no major clinical influence of sarcoidosis on thyroid function and autoimmunity was found, and screening for thyroid disease in such patients does not seem imperative [5].

Our observation illustrates an original case of Graves' disease, the course of which was marked by the onset of active sarcoidosis presented as Löfgren's syndrome complicated by hypercalcemia. Given its rarity and the lack of a causal link, the association between Graves' disease and sarcoidosis may be mere coincidence. More studies could allow us to understand more common etiopathogenic mechanisms, as both are chronic inflammatory pathologies and a common immune mechanism appears to be behind them.

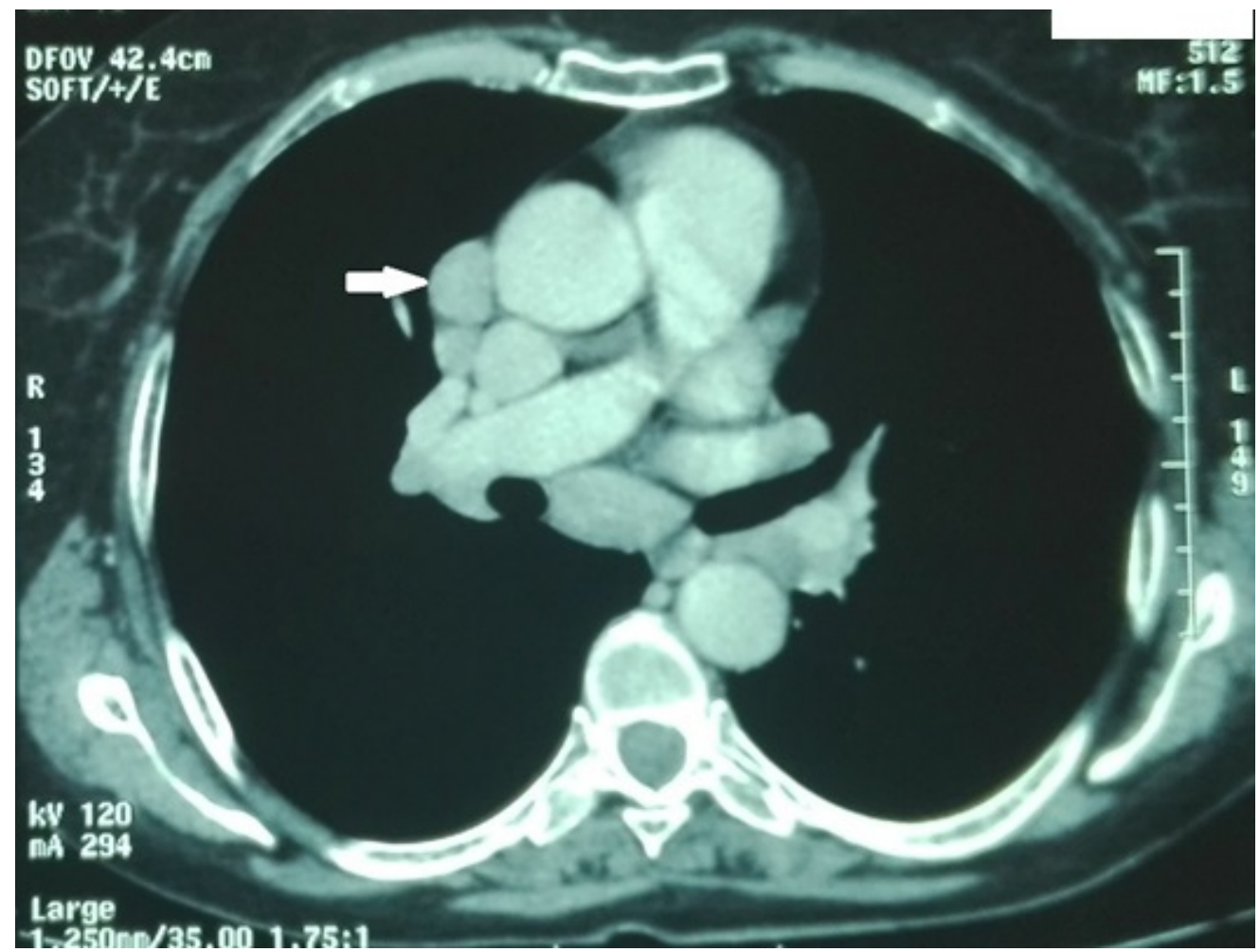

Figure 1: Axial section of a thoracic CT scan with contrast injection showing mediatinal lymphadenopathy (white arrow) in relation to stage I sarcoidosis.

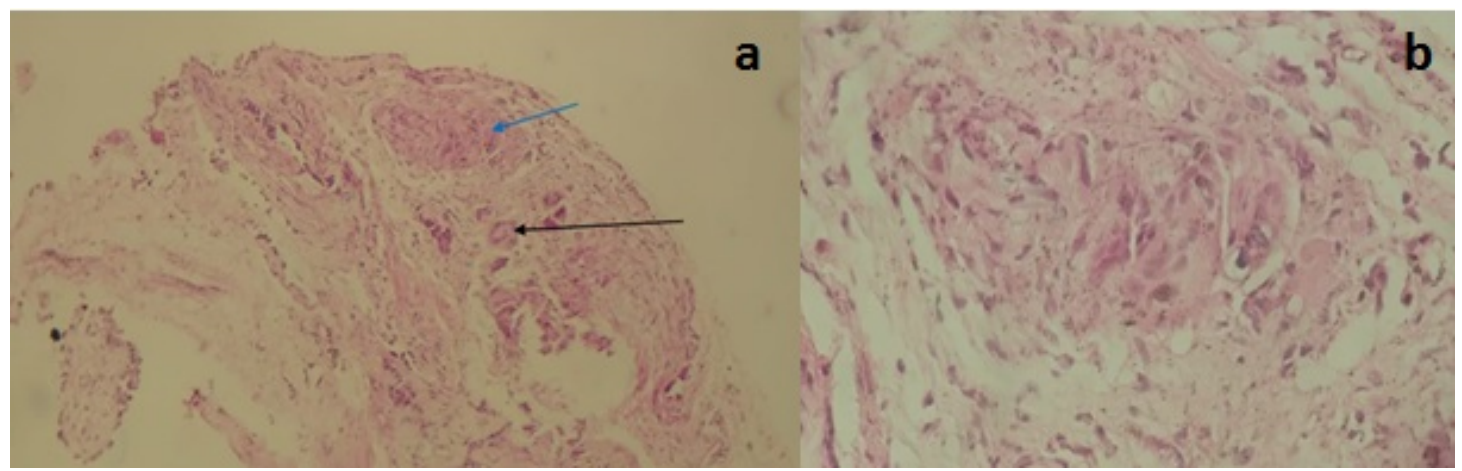

Figure 2: a: Inflammatory granulomatous epithelioid and gigantocellular rearrangement without caseous necrosis. The blue arrow is showing the granuloma, the black arrow is showing giant cells (HE $x$ 100). b: focus on the granuloma (HE x 400) 


\section{Disclosure}

This clinical case was written based on clinical observations and received no funding.

\section{Conflict of interest}

The authors have no conflicts of interest.

\section{References}

1. El Kassimi I, Rkiouak A, Hammi SE, Sekkach Y. Bilateral Exophthalmos caused by Systemic Sarcoidosis: a case report. Eur J Med Case Rep 2018; 2(3):108-10. doi: 10.24911/ejmcr/173-1535106149.

2. Karlish AJ, MacGregor GA. Sarcoidosis, thyroiditis, and Addison's disease. Lancet 1970; 2(7668):330-33. doi: 10.1016/s0140-6736(70)92871-0.

3. Gentilucci UV, Picardi A, Manfiini S, D'Avola D, Costantino S, Pozzilli P. Granulomatous thyroiditis: an unexpected finding leading to the diagnosis of sarcoidosis. Acta Biomed 2004; 75(1):69-73.
4. Nakamura H, Genma R, Mikami T, Kitahara A, Natsume H, Andoh S. et al. High incidence of positive autoantibodies against thyroid peroxidase and thyroglobulin in patients with sarcoidosis. Clin Endocrinol (Oxf) 1997; 46(4):467-72. doi: 10.1046/j.1365-2265.1997.1630976.x.

5. Ilias I, Panoutsopoulos G, Batsakis C, Nikolakakou D, Filippou N, Christakopoulou I. Thyroid function and autoimmunity in sarcoidosis: a case-control study. Croat Med J 1998; 39(4):404-06. 
